\title{
Low-dose radiation therapy: could it be a game-changer for COVID-19?
}

\author{
A. Montero ${ }^{1}\left(\mathbb{D} \cdot\right.$ M. Arenas $^{2} \cdot$ M. Algara $^{3}$
}

Received: 13 April 2020 / Accepted: 12 May 2020 / Published online: 25 May 2020

(c) Federación de Sociedades Españolas de Oncología (FESEO) 2020

It is only afterwards that a new idea seems reasonable.

To begin with, it usually seems unreasonable

-Isaac Asimov -

At the beginning of December 2019, the first cases of a new acute respiratory syndrome, of extraordinary contagiousness, began to be described in the city of Wuhan, the capital of Hubei province, in central China. New virus, a severe acute respiratory syndrome coronavirus 2 (SARS-CoV-2) thus far unknown in the human species, was identified as the causative agent. Since then, humanity has witnessed a rapid spread of this RNA virus throughout the world, being recognized by the World Health Organization (WHO) as a pandemic on March 11, 2020. As of 12th April 2020, over 1614,951 cases and nearly 100,000 deaths are reported worldwide [1]. The consequences of the coronavirus disease (COVID-19) have been quickly noticed, being Spain is one of the countries where the infection has shown the most virulence, putting our national health care system at an imaginable stress and with evident risk of collapse in case of uncontrolled outbreaks.

COVID-19 is a respiratory disease caused by this coronavirus that produces significant morbidity and mortality. The most frequent symptoms are fever, dry cough, asthenia, expectoration, dyspnea, sore throat, headache, arthromyalgia, among others. Some patients develop pneumonia that can lead to respiratory failure or severe acute respiratory syndrome (SARS). According to the Chinese experience,

A. Montero

angel.monteroluis@gmail.com

1 Oncología Radioterápica, Hospital Universitario HM Sanchinarro, Madrid, Spain

2 Oncología Radioterápica, Hospital Universitario Sant Joan de Reus, Universitat Rovira I Virgili, Reus, Spain

3 Oncología Radioterápica, Hospital de la Esperanza, Parc de Salut del Mar, Universitat Autónoma de Barcelona, Barcelona, Spain
$81 \%$ of the clinical pictures were mild in nature with an overall case fatality rate of $2.3 \%$, while a small subgroup of $5 \%$ had respiratory failure, septic shock, and multi-organ failure leading to death in half of these cases. Some patients with COVID-19 disease may experience a cytokine release syndrome (SLC) caused by a systemic inflammatory response that occurs when large numbers of leukocytes (neutrophils, macrophages, and mast cells) are activated and release large amounts of proinflammatory cytokines (interleukin (IL)-6, IL-10, interferon (IFN), monocyte chemoattractant protein-1 (MCP-1), granulocyte-macrophage colony-stimulating factor (GM-CSF), tumor necrosis factor $\alpha$ (TNF- $\alpha$ ), IL-1, IL-2, IL-8). Clinical observations suggest that when the immune response is unable to effectively control the virus, as in older people with a weakened immune system, the virus would spread more efficiently, causing lung tissue damage, which would activate macrophages and granulocytes and would lead to the massive release of proinflammatory cytokines. This pulmonary hyperinflammation would be associated with SARS, which has been described as the main cause of COVID-19 mortality [2]. There are two distinct but overlapping pathological subsets, the first triggered by the virus itself and the second, the host response. Although in the first stage patients will benefit from drug therapy directed against the virus, its usefulness in advanced stages may be questionable. Similarly, the use of anti-inflammatory therapy applied too early may not be necessary and may even cause viral replication. In the second stage of established lung disease, viral multiplication and localized inflammation in the lung is the norm. During this stage, patients develop viral pneumonia, with a cough, fever, and possibly hypoxia, chest radiograph images, or computed tomography with bilateral infiltrates or ground glass opacities. Blood tests reveal an increase in lymphopenia, along with transient elevation of transaminases. Systemic inflammation markers may be elevated, but not markedly. It is at this stage that the majority of COVID-19 patients would need to be hospitalized for close observation and treatment. If hypoxia occurs, patients are likely to progress to requiring mechanical ventilation, and in that situation, the use of anti-inflammatory therapy 
may be helpful and may be used judiciously. A minority of patients with COVID-19 will progress to the third and most severe stage of the disease, manifesting as a syndrome of extra-pulmonary systemic hyperinflammation. At this stage, systemic inflammation markers will be elevated and COVID-19 infection causes a decrease in helper, suppressor, and regulatory T cells. [3].

Currently, there is no effective treatment capable of treating SARS-CoV-2, and the only treatments are those aimed at the side effects caused by the virus, such as inflammation and pulmonary fibrosis, recognized as the first causes of death. Chloroquine/hydroxychloroquine treatment has demonstrated some efficacy for COVID-19. The results of the study by Chen et al. from Wuhan University, showed improvement in those COVID-19 patients who were administered hydroxychloroquine versus placebo in addition to standard treatment with oxygen therapy, antivirals, antibiotics, immunoglobulins, or corticosteroids and also hydroxychloroquine could transmit some protection against worsening of the disease [4]. Likewise, Gautret et al. observed a possible synergistic effect of the combination of hydroxychloroquine and azithromycin, although the authors also warn against a possible unwanted risk effect in relation to the severe prolongation of the QT interval induced by the association of the two drugs [5]. Despite the encouraging results, both studies have limitations in relation to a small sample size, short follow-up, lack of group control and a not inconsiderable percentage of patients abandoned the studies but have established the most widely used treatment today to deal with SARS-CoV-2 infection. However, a recent systematic review by Pacheco and Riera on the efficacy of chloroquine or hydroxychloroquine in COVID-19 patients concluded that according to the data from the two available studies, and of their limited methodological quality, the efficacy and safety of chloroquine or hydroxychloroquine treatment in COVID-19 patients is still uncertain and that its regular use should not be recommended until further evidence is available [6]. Suppression of the proinflammatory members of the IL- 1 and IL-6 family has been shown to have a therapeutic effect in many inflammatory diseases, including viral infections. Suppression of IL-1 $\beta$ by IL-37 in an inflammatory state induced by COVID-19 may have a therapeutic effect in this pathology. Overall, there appear to be some positive results for the use of corticosteroids in viral infections such as SARS-CoV-2. Corticosteroids are used because of their known ability to modulate a variety of involved cytokines (including IL-1, IL-6, IL-8, IL-12, and $\mathrm{TNF} \alpha$ ). Several human studies found that corticosteroid seemed effective in reducing immunopathological damage. Another treatment that has been shown to be effective is the monoclonal anti-human IL-6 receptor antibody, tocilizumab (used in the treatment of rheumatoid arthritis). It can specifically bind the two forms of the IL-receptor 6 (membrane-bound IL-6 receptor (mIL6R) and soluble IL-6 receptor (sIL6R)) and inhibit signal transduction. Russell et al. have recently published a systematic review of current evidence for treatment with immunosuppressants, cytotoxic chemotherapy, steroids, TNF- $\alpha$ blockers, IL- 6 block, Janus kinase inhibitors (JAK), block IL-1, mycophenolate, tacrolimus, anti-CD20 and CTLA4-Ig. After reviewing 89 studies, the authors' conclusion is that low doses of prednisolone and tacrolimus may have beneficial effects on COVID-19, as well as that IL-6 levels are associated with the severity of pulmonary complications, although there is no evidence regarding the beneficial impact of IL-6 inhibitors on the course of COVID-19 disease [7].

In the constant and incessant search for treatments against COVID-19, it has been suggested that low-dose radiation therapy (LD-RT) could play a role for their anti-inflammatory effects. The dose is below $1 \%$ of doses used for cancer treatment and the range between 0.3 and 0.7 Gy. LD-RT has been used for more than a century in the treatment of pneumonia, especially interstitial and atypical. In the review by Calabrese et al., low doses of radiation to the lungs were found to be associated with good response rates and resolution of symptoms. The authors reviewed 15 studies including 863 cases of bacterial pneumonia (lobular and bronchopneumonia), interstitial, and atypical pneumonia that were effectively treated with low-dose X-rays, improving symptoms, increasing cure, and reducing mortality. The mechanism by which X-ray treatment acts on pneumonia involves the induction of an anti-inflammatory phenotype that leads to a rapid reversal of clinical symptoms, facilitating resolution of the disease. Treatment was most effective when irradiation was administered 6-14 days after the clinical onset of the disease. After 14 days, the successful response rate decreased by approximately $50 \%$. The authors' conclusion is that LD-RT offers excellent potential as a treatment for interstitial pneumonia, especially when used during the early stages of the disease [8].

The anti-inflammatory effects of LD-RT have been confirmed in several experimental models, both in vitro and in vivo and in clinical studies. The radiobiological mechanisms that support this claim are increasingly known. Unlike high-dose radiation therapy that induces the production of proinflammatory cytokines in immune and endothelial cells, paradoxically LD-RT (0.5-1.5 Gy) acts on cells involved in the inflammatory response, producing anti-inflammatory effects. The mechanisms that explain these anti-inflammatory effects are due to a decrease in polymorphonuclear cells to endothelial cells and the induction of apoptosis, a decrease in the expression of adhesion molecules (selectins (P-, L-, E-), ICAM, VCAM), a decreased production of nitric oxide (NO), increased activation of nuclear factor kappa-beta (NK$\mathrm{KB}$ ), and increased production of cytokines by endothelial 
cell and immune cells (IL-10, transforming growth factor anti-inflammatory cytokine $\beta 1$ (TGF- $\beta$ 1) ) [9-13]. All of these changes result in a local anti-inflammatory environment that would explain the clinical effects of LD-RT. The evidence obtained from laboratory studies demonstrated the maximum anti-inflammatory effect of radiotherapy in the environment with doses of 0.3-0.7 Gy per fraction [9, 10]. Likewise, in vitro experiments showed that the antiinflammatory effect of LD-RT was greatest at $48 \mathrm{~h}$ after irradiation and was lost after $72 \mathrm{~h}$ justifying the interval of at least $48 \mathrm{~h}$ between the administrations of consecutive radiation therapy fractions [8-13].

Choosing the right time to administer LD-RT in COVID19 patients is challenging. It is at the beginning of the proinflammatory phase that the use of anti-inflammatory treatments such as corticosteroids and cytokine inhibitors tocilizumab (IL-6 inhibitor) or anakinra (IL-1 receptor antagonist) seems to be justified. Presumably, it is in this phase where LD-RT to both lungs could be effective by acting as a powerful anti-inflammatory agent against the cascade of proinflammatory cytokines [2]. There are several advantages associated with the use of LD-RT as proposed: radiotherapy treatment units are available and the procedure for the proposed treatment is optimized to simplify its development as much as possible. Furthermore, the objective of this treatment is pragmatically designed to be used in a segment of patients with limited treatment alternatives and who in the current situation are not candidates for mechanical ventilation measures and intensive care units (ICU). Kirkby and MacKenzie recently suggested that a treatment with LD-RT, from 30 to $100 \mathrm{cGy}$, to the lungs of a patient with COVID-19 pneumonia could reduce inflammation and alleviate the symptoms that life threatening [14].

Although the exact magnitude of the benefit of LD-RT is uncertain, it can be said that the probability that the damage is very low. For reference, a CT scan of the chest is around $5 \mathrm{cGy}$. Therefore, LD-RT therapy would be in the order of 6-10 CT, well below the known threshold for any typical radiation side effect. What is unclear is whether this low dose could adversely modulate the immune environment to exacerbate underlying lung dysfunction, although previously cited laboratory and experimental animal studies have not observed this [8-10]. The safety of LD-RT has been analyzed by different studies that use it for the treatment of benign non-tumor pathology, concluding in all of them that the risk of presenting complications attributable to irradiation is extremely low with the doses suggested in this study [15-18]. Regarding the induction of secondary malignancies, it is added that this risk will be insignificant given the target population of predominantly older patients and the proposed ultra-low dose. Furthermore, secondary malignancies are not considered clinically relevant in this cohort with a high mortality rate a few weeks after infection.
Currently, only ICU admittance can recover patients severely afflicted by COVID pneumonia. Severely diseased COVID-19 patients with pre-existing comorbidities and older patients represent a gap in the current clinical practice because they usually are not considered candidates to aggressive manoeuvres. Ultra LD-RT to both lungs could be an option for these patients with COVID-19 pneumopathy by decreasing the inflammatory storm while contributing to reduce the overload of the health system, especially in ICU. We are convinced that the possibility of having a treatment that is not subject to fluctuations in its acquisition, with low cost and available in many centers without the need for a high financial investment should also be considered under the current circumstances of the COVID-19 pandemic.

\section{Compliance with ethical standards}

Conflict of interest The authors have declared no conflicts of interest.

Ethical approval This article do not contain any studies with human participants or animals performed by any of the authors.

Informed consent For this type of study formal consent is not required.

\section{References}

1. https://www.who.int/emergencies/diseases/novel-coronaviru s-2019

2. Conti P, Ronconi G, Caraffa A, Gallenga CE, Ross R, Frydas I, Kritas SK. Induction of pro-inflammatory cytokines (IL-1 and IL-6) and lung inflammation by coronavirus-19 (COVI-19 or SARS-CoV-2): anti-inflammatory strategies. J BiolRegul Homeost Agents. 2020. https://doi.org/10.23812/CONTI-E.

3. Siddiqui HK, Mehra MR. Illness in native and immunosuppressed states: a clinical-therapeutic staging proposal. J Heart Lung Transplantation COVID-19

4. Chen J, Liu D, Li L, Liu P, Xu Q, Xia L, et al. A pilot study of hydroxychloroquine in treatment of patients with common coronavirus disease-19 (COVID-19). J Zhejiang Univ (MedSci). 2020. https://doi.org/10.3785/j.issn.1008-9292.2020.03.03.

5. Gautret P, Lagier JC, Parola P, et al. Hydroxychloroquine and azithromycin as a treatment of COVID-19: results of an openlabel non-randomized clinical trial. Int J Antimicrob Agents. 2020. https://doi.org/10.1016/j.ijantimicag.2020.105949.

6. Pacheco R, Riera R. Hydroxychloroquine and chloroquine for COVID-19 infection. Rapid systematic review. J Evid-Based Healthcare. 2020. https://doi.org/10.17267/2675-021Xeviden ce.v2i1.2843.

7. Russell B, Moss C, George G, et al. Associations between immunosuppressive and stimulant drugs and the novel systematic review COVID-19-a of current evidence. Ecancermedical science. 2020;14:1022.

8. Calabrese EJ, Dhawan G. How radiotherapy was historically used to treat pneumonia: could it be useful today? Yale J Biol Med. 2013;86(4):555-70.

9. Rödel F, Frey B, Manda K, Hildebrandt G, Hehlgans S, Keilholz L, Rödel C. Immunomodulatory properties and molecular effects 
in inflammatory diseases of low-dose $\mathrm{x}$-irradiation. Front Oncol. 2012;2:120.

10. Arenas M, Sabater S, Hernández V, Rovirosa A, Lara PC, Biete A, Panés J. Anti-inflammatory effects of low-dose radiotherapy. Strahlenther Onkol. 2012;188(11):975-81.

11. Arenas M, Gil F, Gironella M, Hernández V, Biete A, Piqué JM, Panés J. Time course of anti-inflammatory effect of low-dose radiotherapy: correlation with TGF-beta(1) expression. Radiother Oncol. 2008;86(3):399-406 Epub 2007 Nov 26.

12. Lödermann B, Wunderlich R, Frey S, Schorn C, Stangl S, Rödel F, Keilholz L, Fietkau R, Gaipl US, Frey B. Low dose ionising radiation leads to a NF- $\mathrm{KB}$ dependent decreased secretion of active IL- $1 \beta$ by activated macrophages with a discontinuous dosedependency. Int J Radiat Biol. 2012;88(10):727-34.

13. Torres Royo L, Antelo Redondo G, ÁrquezPianetta M, Arenas PM. Low-dose radiation therapy for benign pathologies. Rep Pract Oncol Radiother. 2020;25(2):250-4. https://doi.org/10.1016/j. rpor.2020.02.004.

14. Kirkby C, Mackenzie M. Is low dose radiation therapy a potential treatment for COVID-19 pneumonia? Radiother Oncol. 2020. https://doi.org/10.1016/j.radonc.2020.04.004.
15. Jansen JTM, Broerse JJ, Zoetelief J, Klein C, Seegenschmiedt HM. Estimation of the carcinogenic risk of radiotherapy of benign diseases from shoulder to heel. Radiother Oncol. 2005;76(3):270-7.

16. Trott KR, Kamprad F. Estimation of cancer risks from radiotherapy of benign diseases. Strahlenther Onkol. 2006;182(8):431-6.

17. Mazonakis M, Damilakis J. Cancer risk after radiotherapy for benign diseases. Phys Med Eur J Med Phys. 2017;42:285-91.

18. Trott MR, McKeown SR, Hatfield P, Prestwich RJD, Shaffer RE. Taylor RE Radiotherapy for benign disease; assessing the risk of radiation-induced cancer following exposure to intermediate dose radiation. Br J Radiol. 2015;88:20150405.

Publisher's Note Springer Nature remains neutral with regard to jurisdictional claims in published maps and institutional affiliations. 